\title{
Repercussões da pandemia SARS-CoV-2 para estudantes de medicina do Ceará -
}

\section{Brasil}

\author{
Repercussions of the SARS-CoV-2 pandemic for medical students in Ceará - Brazil \\ Repercusiones de la pandemia de SARS-CoV-2 en los estudiantes de medicina de Ceará - Brasil
}

Recebido: 30/01/2022 | Revisado: 04/02/2022 | Aceito: 09/02/2022 | Publicado: 14/02/2022

Bárbara Calisto Campos

ORCID: https://orcid.org/0000-0002-9100-508X

Centro Universitário Christus, Brasil

E-mail: barbaracalisto09@gmail.com

Marina Leite de Oliveira

ORCID: https://orcid.org/0000-0001-8554-2316

Centro Universitário Christus, Brasil

Email: marinaleiteoliveiraa@gmail.com

Thaine Mirla Rocha

ORCID: https://orcid.org/0000-0001-7701-7140 Centro Universitário Christus, Brasil

E-mail: thainemirla@gmail.com

Laís Rêgo Borges

ORCID: https://orcid.org/0000-0002-1878-8970 Centro Universitário Christus, Brasil E-mail: laisregoborgess@gmail.com

Beatriz Calisto Campos

ORCID: https://orcid.org/0000-0002-3087-3086 Centro Universitário Christus, Brasil

E-mail: biiiacalisto26@gmail.com

Lucas Fortaleza de Aquino Ferreira

ORCID: https://orcid.org/0000-0001-8815-7309 Centro Universitário Christus, Brasil Email: lucas_fortaleza@hotmail.com

Joyce Rodrigues Façanha

ORCID: https://orcid.org/0000-0002-7511-6345 Centro Universitário Christus, Brasil

Email: joyce.facanha@unichristus.edu.br

Arnaldo Aires Peixoto Junior

ORCID: https://orcid.org/0000-0001-6225-934X Centro Universitário Christus, Brasil

E-mail: arnaldoapj@gmail.com

Raquel Autran Coelho Peixoto

ORCID: https://orcid.org/0000-0002-2998-2779

Centro Universitário Christus, Brasil

E-mail: raquelautrancp@gmail.com

\section{Resumo}

Objetivos: Avaliar repercussões psicológicas e a percepção de preparo para atuar no atendimento a pacientes na pandemia de COVID-19 entre estudantes de medicina de Fortaleza-CE, Brasil. Metodologia: Trata-se de estudo transversal, analítico, de dezembro de 2020 até fevereiro de 2021. Estudantes de medicina do pré-internato e internato de três escolas médicas brasileiras preencheram voluntariamente um questionário online sobre dados sociodemográficos, percepção de risco pela pandemia COVID-19 e sintomas de estresse pós-traumático avaliados pela Impact of Event Scale (IES) em sua versão brasileira validada. Todos assinaram termo de consentimento livre e esclarecido. $\mathrm{O}$ estudo foi aprovado no Comitê de Ética e Pesquisa das Instituições. Ao comparar grupos, utilizou-se testes de Spearman e Kruskal Wallis, com erro alfa menor que 5\%. Resultados: 94 estudantes participaram, sendo 60,6\% do sexo feminino e 86,2\% de universidades particulares. 53,3\% tinham percepção de alto risco de contágio com o vírus e 40,2\% relataram qualidade de sono insatisfatória. A pontuação média da IES dos participantes foi 14,8 +/- 5,78, demonstrando que houve um impacto estressante. Houve associação do escore da IES com percepção do risco de contágio, qualidade do sono, tipo de universidade e acompanhamento psicológico. Conclusão: A pandemia do Covid-19 gerou impacto estressante nos estudantes de medicina avaliados, com associação significante com percepção do risco de contágio, qualidade do sono, tipo de universidade e acompanhamento psicológico. Esses fatores são importantes para futuras intervenções psicológicas entre estudantes.

Palavras-chave: COVID-19; Internato e residência; Educação à distância; Transtornos de Estresse pós-traumáticos. 


\begin{abstract}
Objectives: To assess psychological repercussions and the perception to act in patient care in the Covid-19 pandemic among medical students in Fortaleza-CE, Brazil. Methodology: This was a cross-sectional, analytical study from December 2020 to February 2021. Participants were pre-internship and internship medical students from three Brazilian medical schools and voluntarily completed an online questionnaire about sociodemographic data, perceived risk for the COVID-19 pandemic, and post-traumatic stress symptoms assessed by the Impact of Event Scale (IES) in its Brazilian version. All of them signed an informed consent form, and the study was approved by the Ethics and Research Committee of the institutions. Spearman and Kruskal Wallis tests were used to compare the groups, with alpha error lower than $5 \%$. Results: There were 94 participants, $60.6 \%$ female, $86.2 \%$ from private universities. 53.3\% had a perception of high risk of infection with the virus and $40.2 \%$ reported unsatisfactory sleep quality. The mean IES score of the participants was $14.8+/-5.78$, showing that there was a stressful impact. There was statistical significance related to the IES scores when assessing risk of infection, sleep, university, and psychological follow-up. Conclusion: The Covid-19 pandemic generated a stressful impact on the medical students evaluated, with importance for future psychological interventions in times of pandemics.
\end{abstract}

Keywords: COVID-19; Internship and residency; Education distance; Stress disorders, post-traumatic.

\title{
Resumen
}

Objetivos: Evaluar las repercusiones psicológicas y la percepción de la preparación en la atención de pacientes en la pandemia de COVID-19 entre los estudiantes de medicina de Fortaleza-CE, Brasil. Métodos: Un estudio transversal y analítico desde diciembre de 2020 hasta febrero de 2021. Los participantes fueron estudiantes de medicina de preinternado y de internado de tres facultades de medicina brasileñas y completaron voluntariamente un cuestionario en línea sobre datos sociodemográficos, percepción de riesgo por la pandemia COVID-19 y síntomas de estrés postraumático evaluados por la Escala de Impacto del Evento (IES) en su versión brasileña. Todos firmaron un formulario de consentimiento informado y el estudio fue aprobado por el Comité de Ética e Investigación de las instituciones. Se utilizaron las pruebas de Spearman y Kruskal Wallis para comparar los grupos, con un error alfa inferior al 5\%. Resultados: Participaron 94 estudiantes, 60,6\% eran mujeres, 86,2\% procedían de universidades privadas. 53,3\% evaluó un alto riesgo de infección por el virus y el 40,2\% declaró una calidad de sueño insatisfactoria. La puntuación media del IES de los participantes fue 14,8 +/- 5,78, lo que demuestra que hubo un impacto estresante. Hubo significación estadística relacionada con las puntuaciones del IES al evaluar el riesgo de contagio, el sueño, la universidad y el seguimiento psicológico. Conclusión: La pandemia de Covid-19 generó un impacto estresante en los estudiantes de medicina evaluados, con importancia para futuras intervenciones psicológicas en las pandemias.

Palabras clave: COVID-19; Internado y residencia; Educacíon a distancia; Trastornos por estrés postraumático.

\section{Introdução}

A pandemia pela Covid-19, causada pelo vírus SARS-CoV-2, teve seu primeiro caso registrado no Brasil no dia 29 de fevereiro de 2020, tendo sido confirmada sua transmissão em todas as regiões brasileiras em meados de maio do mesmo ano. As autoridades governamentais implementaram medidas de distanciamento social, incluindo o fechamento de escolas e faculdades como meio de conter a transmissão interpessoal e propagação do vírus. (Goularte et al, 2021; Khan et al., 2020).

As instituições de ensino superior precisaram se adequar à nova demanda, com implementação do ensino remoto em circunstâncias pouco favoráveis, tornando necessário qualificar os docentes, assim como aderir novas formas de ensino. (Kubrusly et al., 2021; Rose, 2020). Algumas faculdades de Medicina interromperam, inclusive, as atividades práticas em hospitais de ensino vinculados, assim como no próprio campus, uma vez que os alunos poderiam adquirir a doença ou transmiti-la, mesmo assintomáticos (Tolsgaard et al., 2020).

Estudos sugerem que emergências de saúde pública, como epidemias, podem ter muitos efeitos psicológicos sobre estudantes universitários, que podem ser expressos como ansiedade e preocupação. (Song et al., 2019). A ansiedade pode também ser atribuída ou agravada pelo isolamento social, que ocorreu em muitos países com transmissão comunitária sustentada, como o Brasil (Cao et al., 2020).

Estratégias para auxiliar os estudantes a lidar com seus sentimentos em épocas de crise, como epidemias, tem sido um desafio para as universidades (Cao et al., 2020; Khan et al., 2020). Na literatura, observou-se que cerca de $80 \%$ de jovens dos avaliados em um estudo tiveram seus problemas relacionados à saúde mental exacerbados com a suspensão das atividades educacionais, assim como, com a diminuição da interação social do cotidiano no ambiente escolar (Khan et al., 2020). 
Há algumas escalas validadas para tentar quantificar o impacto de eventos estressantes sobre indivíduos. Uma das mais utilizadas tem sido a escala Impact of Event Scale (IES). Cerca de 51\% dos estudantes universitários entrevistados por meio da escala IES tiveram pontuação moderada a grave com relação ao impacto psicológico do surto pela COVID-19 (OdriozolaGonzález et al., 2020). Um estudo com resultados semelhantes, realizado na China, também no período da pandemia, utilizou o IES, e a maioria dos participantes classificou o impacto psicológico, como moderado a grave, sendo o gênero feminino e o status de aluno associados com o maior nível de impacto (Khan et al., 2020; Wang et al., 2020).

O uso de escala de avaliação de impacto de evento estressante validada em outros países nesse momento de pandemia pela COVID-19 validada poderá auxiliar no monitoramento da saúde mental dos estudantes, podendo ser útil para apoiar estratégias de apoio nas universidades. O presente estudo objetivou avaliar as repercussões psicológicas durante a pandemia de Covid-19 para os estudantes de medicina, bem como sua percepção de preparo para atuar no atendimento a pacientes com síndromes gripais.

\section{Metodologia}

Trata-se de estudo observacional transversal, analítico, com abordagem quantitativa, com estudantes do curso de graduação de Medicina no período do internato ou pré-internato de duas instituições de ensino superior privadas e uma instituição de ensino superior pública do estado do Ceará-Brasil, no período de dezembro de 2020 a fevereiro de 2021 (Capp et al., 2020).

Neste período, as Instituições de Ensino Superior haviam migrado para ensino remoto exclusivo, quando estavam em lockdown no estado. Então, o plano de ensino teórico foi aplicado por meio das plataformas digitais de ensino. Nenhum aluno havia sido vacinado. Todos os participantes estavam matriculados no período no qual se voluntariaram, por amostragem aleatória.

Foram coletadas, por meio de questionário online autoaplicado, informações demográficas para caracterização dos perfis da amostra de internos, como idade, sexo, formação prévia, período matriculado, hábitos de vida, doenças clínicas e psiquícas já diagnosticadas, medicamentos de uso contínuo e repercussões acadêmicas e psicológicas da crise mundial devido à pandemia de covid-19. O convite para participar do estudo foi feito por meio de um link de formulário eletrônico no formato Google forms, enviado por whatsapp em grupos de alunos das escolas avaliadas.

Os itens avaliaram as percepções dos alunos sobre repercussões da pandemia de covid-19 em suas vidas pessoal e acadêmica. As informações pessoais dos entrevistados foram tornadas anônimas para manter e proteger a confidencialidade.

Usou-se a escala Impact of Event Scale (IES) em sua versão brasileira, que possibilitou a avaliação do impacto psicológico (Silva et al., 2010; Guanilo et al., 2005; Horowitz et al., 1979). Trata-se de uma escala de 15 itens, que mostrou ser importante para validação de estresse em surtos virais (Vanaken et al., 2020), como escala de Likert de quatro pontos, tendo um subconjunto de itens de inclusão $(1,4,5,6,10,11$ e 14) e um outro subconjunto com os itens restantes relacionados à evitação $(2,3,7,8,9,12,13$ e 15), que foram utilizados para avaliar o impacto do estresse traumático, que tenha gerado ansiedade excessiva e memórias angustiantes relacionadas a determinada experiência que ocorreu na pandemia do Covid-19. A resposta de cada item pode ter pontuação zero (nunca), para endosso negativo, e 1 (raramente), 3 (às vezes) e 5 (frequentemente), para os três graus de endosso positivo para intensidade e frequência. A soma das pontuações varia de 0 a 75 pontos, de tal forma que, quanto maior for a pontuação atingida, maior o impacto psicológico. A interpretação das pontuações: 0-8 pontos: nenhum impacto significativo; 9-25 pontos: evento com impacto; 26-43: evento de impacto poderoso; 44-75: evento de impacto severo, sendo capaz de alterar a capacidade funcional do paciente. Para pontuações > 27 pontos, infere-se um maior risco de TEPT (Transtorno de Estresse Pós-Traumático), indicando a busca para um profissional da saúde em soma maior ou igual a 35 pontos, sendo o melhor ponto de corte para um provável diagnóstico de TEPT (Quadro 1) (Prashanth et al., 2015; Zhang \& Ma, 2020). 
Silva et al. (2010) adaptaram e traduziram semanticamente para o português brasileiro, mantendo as características fundamentais da escala original de Horowitz et al. (1979) com algumas alterações em relação à primeira tradução nos itens 1, $3,4,10,11,12$ e 15, para melhor entendimento de determinadas expressões, evitando-se dúvidas.

Quadro 1 - Interpretação das pontuações da escala IES.

\begin{tabular}{l|c}
\hline \multicolumn{1}{c|}{ Interpretação } & Escore (pontos) \\
\hline Nenhum impacto significativo & 0 a 8 \\
Evento com impacto & 9 a 25 \\
Evento de impacto poderoso & 26 a 43 \\
Evento de impacto severo, & \\
capaz de alterar capacidade funcional & 44 a 75 \\
\hline
\end{tabular}

Fonte: Autores (2022).

As respostas obtidas foram organizadas em planilha no programa Microsoft Excel 2003. Foram realizadas análises estatísticas descritivas (percentual e média). Posteriormente, as respostas foram transferidas para o Jamovi (versão 1.6.23.0) para análise de dados. Foram utilizados os testes de qui-quadrado de associação, e teste de Spearman e Kruskal Wallis para a comparação dos grupos, considerando-se um erro alfa menor que 5\%.

O trabalho foi aprovado no Comitê de Ética em Pesquisa da instituição, com anuência das instituições de ensino envolvidas. Todos os participantes assinaram eletronicamente o Termo de Consentimento Livre e Esclarecido para participar do estudo e tinham idade maior de 18 anos.

\section{Resultados}

Foram incluídos 94 alunos do curso de Medicina, sendo 13 alunos de escolas públicas e 81 matriculados em instituições privadas. $83 \%$ estavam no período do internato e o restante (17\%) correspondiam ao pré-internato. A média de idade foi de 23 anos, com desvio padrão de 2,4. Da população estudada, 60,6\% eram do sexo feminino, 79,8\% eram solteiros, $75,5 \%$ moravam com os pais. Dos avaliados, 53,2\% apresentavam uma percepção de alto risco de se contaminar com o vírus da Covid-19. Da amostra avaliada, 63,8\% acreditavam que a forma de utilização dos EPIs, tanto a paramentação quanto a desparamentação, era satisfatória. Dos alunos participantes, 42,6\% mostraram insatisfação com a qualidade do sono, e 36,8\% não praticavam atividade física. (Tabela 1). 
Research, Society and Development, v. 11, n. 3, e10811326341, 2022

(CC BY 4.0) | ISSN 2525-3409 | DOI: http://dx.doi.org/10.33448/rsd-v11i3.26341

Tabela 1: Dados clínico-demográficos da população estudada $(\mathrm{N}=94)$.

\begin{tabular}{|c|c|c|c|}
\hline Característica & Tipo & $\mathbf{N}$ & $\%$ \\
\hline \multirow[t]{2}{*}{ Estado civil } & Solteiro & 75 & 79,8 \\
\hline & Casados/união & 17 & 18,1 \\
\hline \multirow[t]{2}{*}{ Gênero } & Feminino & 57 & 60,6 \\
\hline & Masculino & 36 & 38,3 \\
\hline \multirow[t]{5}{*}{ Semestre } & Nono & 12 & 12,8 \\
\hline & Décimo & 48 & 51,1 \\
\hline & Décimo primeiro & 3 & 3,2 \\
\hline & Décimo segundo & 15 & 16 \\
\hline & Pré-internato & 16 & 17 \\
\hline \multirow[t]{2}{*}{ Universidade } & Privada & 81 & 86,2 \\
\hline & Pública & 13 & 13,8 \\
\hline \multirow[t]{2}{*}{ Doença Pisíquica } & Sim & 29 & 30,9 \\
\hline & Não & 65 & 69,1 \\
\hline \multirow{2}{*}{$\begin{array}{l}\text { Acompanhamento com } \\
\text { psicólogo }\end{array}$} & Sim & 33 & 35,1 \\
\hline & Não & 61 & 64,9 \\
\hline \multirow[t]{3}{*}{ Percepção de risco } & Alto & 50 & 53,2 \\
\hline & Médio & 37 & 39,4 \\
\hline & Baixo & 7 & 7,4 \\
\hline \multirow[t]{4}{*}{ Apto para EPI } & Satisfatório & 60 & 63,8 \\
\hline & Muito satisfatório & 6 & 6,4 \\
\hline & Parcialmente insatisfatório & 26 & 27,7 \\
\hline & Muito insatisfatório & 2 & 2,1 \\
\hline \multirow[t]{4}{*}{ Sono } & Satisfatório & 40 & 42,6 \\
\hline & Muito satisfatório & 14 & 14,9 \\
\hline & Parcialmente insatisfatório & 29 & 30,9 \\
\hline & Muito insatisfatório & 11 & 11,7 \\
\hline
\end{tabular}

Fonte: Autores (2022).

Quanto à pontuação na escala IES, a média de pontuação dos alunos foi de 14,8\% (mais ou menos 5,78). Na comparação de grupos, ao comparar a pontuação da IES com os dados clínico-demográficos, com o Teste de Kruskal-Wallis, obteve-se risco de contágio ( $\mathrm{p}=0,03)$, universidade ( $\mathrm{p}=0,034)$, acompanhamento psicológico $(\mathrm{p}=0,05)$ e sono $(\mathrm{p}=0,042)$, idade $(\mathrm{p}=0,424)$, estado civil $(\mathrm{p}=0,199)$, meio de transporte $(\mathrm{p}=0,85)$, horas de lazer $(\mathrm{p}=0,24)$, atividade física $(\mathrm{p}=0,95)$, uso de medicações $(\mathrm{p}=0,164)$, doença psíquica $(\mathrm{p}=0,169)$, doença clínica $(\mathrm{p}=0,199)$, EPI $(\mathrm{p}=0,496)$, frequência de saídas $(\mathrm{p}=$ $0,791)$, aptidão para e concentração nos estudos $(\mathrm{p}=0,981)$ quando comparados com a escala IES. (Tabela 2). 
Tabela 2: Associação da pontuação na Escala de Impacto de Evento (IES) com Idade, Risco de contágio, Preparo para usar EPI, Sono e Concentração nos estudos.

\begin{tabular}{|c|c|c|c|c|c|}
\hline & Idade & $\begin{array}{l}\text { Risco de } \\
\text { contágio }\end{array}$ & $\begin{array}{c}\text { Preparo } \\
\text { para usar } \\
\text { EPI }\end{array}$ & Sono & $\begin{array}{l}\text { Concentração } \\
\text { nos estudos }\end{array}$ \\
\hline IES p & $0.424^{\mathrm{a}}$ & $0.03^{a^{*}}$ & $0.496^{\mathrm{a}}$ & $0.042^{\mathrm{a}^{*}}$ & $0.981^{\mathrm{a}}$ \\
\hline
\end{tabular}

$\mathrm{a}=$ Teste de Kruskal-Wallis. ${ }^{\mathrm{a}^{*}}=$ Diferença estatística. Fonte: Autores (2022).

O Teste de Spearman foi utilizado para avaliar associação da pontuação na Escala de Impacto de Evento (IES) com dados clínicos, sendo observado que não houve associação do escore IES com idade, percepção de proteção profissional, sono e concentração de estudos. Houve, portanto, apenas diferença estatística com a variável Risco de contágio, com p-valor de 0,030 .

\section{Discussão}

Os achados desse estudo demonstraram que o evento da Covid-19 levou a um impacto psicológico nos participantes avaliados, de maneira geral, com pontuação média de 14,8 pontos na escala IES. Em estudos da China, a maioria dos trabalhos apresentou pior impacto psicológico, com escores gerais da IES-R maiores de 24 pontos (Tee et al., 2020). O trabalho foi realizado no período referente à segunda onda de casos da pandemia do SARS-CoV-2. Acredita-se que, por isso, esse evento não tenha se mostrado como causa de um impacto moderado a severo. Além disso, a maioria dos discentes avaliados estavam no período do internato, onde, segundo Barbosa et al. (2015), os alunos costumam ter uma redução de estresse em comparação aos semestres anteriores, pois solidificam seus conhecimentos prévios na prática médica e desenvolvem maior confiança ao lidar com as situações que antes eram novidade.

Os achados do estudo mostraram que a maioria dos estudantes são do sexo feminino e estão matriculados em Instituições de Ensino privadas. Isso vai de acordo com o atual panorama dos médicos recém-formados sendo a maioria do sexo feminino, com 57,4\% daqueles com até 29 anos. (Do Rego et al., 2019). Todos os participantes da pesquisa eram estudantes universitários de escolas médicas. Em um estudo recente, os universitários tiveram a saúde mental significativamente afetada durante a pandemia, em relação a outras categorias ocupacionais. (Jassim et al., 2021).

O fato de grande parte dos estudantes universitários deste trabalho terem sido de escolas médicas particulares pode ter sido um fator de risco para ansiedade neste grupo, uma vez que as repercussões econômicas da pandemia do Covid-19 podem ter comprometido a renda de muitas famílias brasileiras. Em Cao et al. (2020), que utilizou a Escala de Ansiedade Generalizada (GAD-7), em estudantes chineses de medicina, revelou que os efeitos econômicos, cotidianos e atrasos nas atividades acadêmicas foram associados a sintomas de ansiedade de forma positiva.

Segundo Teixeira et al. (2021), ser do sexo feminino e ter o estado civil solteiro são considerados fatores de risco para o sofrimento psíquico. A amostra do estudo em discussão vai de acordo com Teixeira et al. (2021), com a maioria dos alunos sendo solteiros, cerca de $80 \%$. Essa associação é explicada pelo casamento e união estável proporcionarem segurança. Além do gênero e do estado civil, o ensino remoto também foi considerado um fator de risco para o desenvolvimento de algum dano psíquico. Também em Teixeira et al. (2021), cerca de 77\% dos discentes estavam no ensino de educação a distância (EAD) e a maioria $(76 \%)$ relataram ter prejuízo na concentração nos estudos, o que é semelhante ao encontrado na amostra avaliada, com uma parcela de $63 \%$ dos avaliados relatando que sua concentração nos estudos era insatisfatória. 
Os estudantes de medicina estão expostos a infecções transmitidas nos ambientes hospitalares, assim como os profissionais da saúde, sendo importante, portanto, avaliar as suas percepções de risco e o nível de ansiedade relacionados a isso (Torun \& Torun, 2020). Os resultados mostraram que o risco de se contagiar esteve relacionado com mais escores do IES. Nos entrevistados, 53,2 \% dos alunos relataram uma percepção de alto risco de contágio com o vírus e 39,4\% com risco de infecção intermediária (p-valor de 0,03). Em Taghrir et al.(2020), tanto os alunos no pré-internato, como no internato, tiveram altas pontuações nos escores da escala IES-R utilizada. No entanto, ao relacionar os estudantes que estavam na parte teórica do curso de medicina com aqueles dos últimos anos do curso, equivalente aos estágios clínicos, viu-se que as pontuações obtidas pela IES-R relacionadas à ansiedade ao se infectar com COVID-19 no primeiro grupo foram maiores do que os escores obtidos daqueles que estavam no segundo grupo, atribuindo-se ao baixo nível de conhecimento dos alunos que estavam no início da faculdade.

Como aponta a literatura, o risco de contágio é um importante agente estressor e tem risco de desencadear diversas doenças de cunho psicológico, como ansiedade e depressão (Brooks et al., 2020, Fellipe et al., 2021). Este estudo vai de acordo com Felippe et al. (2021), uma vez que em ambos a maioria dos alunos participantes relataram medo frequente de se contaminar com o vírus, mesmo com uso adequado de EPI's. Na amostra do estudo em discussão, cerca de $60 \%$ dos alunos disseram ter aptidão no manejo de EPI's.

O resultado deste estudo, mostrou que $42,6 \%$ dos alunos tiveram, ainda, o sono prejudicado, sendo cerca de $31 \%$ com um sono parcialmente insatisfatório e cerca de $11 \%$ com um sono muito insatisfatório, enquanto 58\% mostraram ser satisfatório. $\mathrm{O}$ sono foi associado a um impacto negativo na saúde mental $(\mathrm{p}=0,042)$. Isso foi semelhante ao encontrado em uma pesquisa realizada na Turquia com estudantes de medicina. Nela, utilizou-se a escala Impact of Events Scale- revised (IES-R) de 22 itens de avaliação durante a pandemia do Covid-19, com 33\% dos alunos referindo que a qualidade do sono estava prejudicada, em relação ao período pré-pandemia (Torun \& Torun, 2020). Ao se avaliar a população geral em um estudo brasileiro, cerca de 55\% das pessoas avaliadas por meio de escalas, como a utilizada em Torun \& Torun (2020), relataram ter o sono comprometido durante a pandemia do SARS-CoV-2 (Goularte et al., 2021). O fato de a maioria dos alunos do estudo terem avaliado o sono como satisfatório pode ser devido à diminuição da carga horária, como ocorreu em alguns cursos das áreas da saúde (Barros et al., 2021). Em Amorim et al. (2018), o sono prejudicado relatado por estudantes de medicina pode ser devido ao pouco horário disponível de estudo, fazendo com que o aluno estude no período da noite. Cabe ressaltar que a diminuição da carga horária também tem relação com o desenvolvimento e agravamento de doenças mentais (Barros et al., 2021).

Na revisão sistemática de Deng et al. (2021), cerca de 33\% dos estudantes universitários chineses apresentaram distúrbios do sono, em relação ao período pré-pandêmico. Em Amorim et al. (2018), sabe-se que os estudantes de medicina já apresentavam dificuldade com o sono antes da pandemia do Covid-19, com $72 \%$ dos entrevistados relatando má qualidade do sono. A importância de se investigar a qualidade do sono é que os problemas para adormecer estão relacionados com dificuldades na concentração e interferem no funcionamento global dos estudantes, além de terem relação com os altos níveis de estresse a que os alunos de medicina são expostos.

$\mathrm{Na}$ amostra do estudo, cerca de $65 \%$ dos alunos avaliados não tinham acompanhamento psicológico e isso foi atribuído a maiores pontuações do IES ( $p=0,05)$. Esse resultado foi semelhante ao estudo de Cardoso et al. (2015), no qual, dos 72 estudantes de medicina de uma Universidade do Rio Grande do Norte, 58 nunca haviam se submetido a um acompanhamento com psicólogo ou com psiquiatra.

Segundo Ornell Felipe et al. (2020), tendo em vista estudos de epidemias anteriores, o número de pessoas com a saúde mental prejudicada será maior que a totalidade das pessoas infectadas pelo vírus, por isso tem crescido a preocupação e o 
interesse na saúde mental dos estudantes de escolas médicas, que repercute na formação dos futuros médicos (Brooks et al., 2020).

Ainda em relação aos estudantes avaliados, a maioria morava com os pais ou com o cônjuge, o que pode ser considerado um fator de proteção ao minimizar o distanciamento social. O isolamento social que ocorre ao se abdicar do convívio com familiares e amigos é um estigma para alunos de medicina que pode ser justificado pela grande demanda de carga horária do curso e por ser o primeiro contato com o processo de doença e morte, quando estes estão nos campos de prática. (Amorim et al., 2018, Barbosa et al., 2015). Segundo Brooks et al. (2020), em uma revisão sistemática, alguns estudos mostraram que os profissionais da saúde ficaram mais expostos ao desenvolvimento de distanciamento social e ansiedade, ao compará-los com a população geral, ambos os grupos em quarentena, obtendo sintomas mais graves de estresse pós-traumático.

A maioria dos pesquisados também saíam de casa mais de três vezes por semana. Isso pode ser atribuído a maioria dos participantes estarem no período do internato e necessitarem ir às suas atividades em hospitais e unidades de saúde para cumprirem a carga horária exigida por suas escolas médicas. Segundo Felippe et al. (2021), existe uma precariedade na disponibilização dos EPIs, assim como, há poucas melhorias nas condições de trabalho para os profissionais da saúde, sendo fatores estressores para os discentes que precisam cumprir as exigências de carga horária nestes locais. Além disso, na pandemia do Covid-19, houve um prejuízo no processo de ensino-aprendizagem, gerando incerteza e preocupação aos estudantes, como cancelamento de atividades ambulatoriais e esgotamento dos profissionais da saúde (Freitas et al., 2021). Em contrapartida, os alunos foram privados da convivência e consolidação das relações formais e informais, mesmo aqueles que relataram sair diariamente, por haver medidas governamentais de restrições para o controle da infecção, o que está relacionado ao desenvolvimento de danos psicológicos (Barros et al., 2021; Deng et al., 2021).

Este estudo apresentou alguns pontos fortes, como ter sido o primeiro estudo a avaliar o impacto psicológico da pandemia do Covid-19 por meio de uma escala validada de impacto de estresse pós-traumático para estudantes de medicina em instituições de ensino superior públicas e privadas de Fortaleza- Ceará.

Como limitações, trata-se de um estudo transversal com número limitado de alunos de uma capital do Brasil. Estudos longitudinais são necessários para avaliar o impacto psicológico, a longo prazo, dos participantes. Foram estudados somente alunos do curso de medicina, não se podendo comparar essa amostra com outros cursos. Além disso, não foram especificados os semestres dos alunos que responderam os questionários no grupo de pré-internato. Dessa forma, não se pôde averiguar a diferença entre alunos do primeiro ano e do último ano do curso. Ademais, outro fator limitante é que não houve grupo controle, portanto, as associações não implicam em causalidade. É possível que tenha havido um viés de seleção, tendo em vista que a maioria dos alunos avaliados residiam no Ceará, que é o estado de residência dos autores do trabalho.

No entanto, apesar das limitações apresentadas pelo estudo, permite-se destacar que apesar da existência de diversos estudos e do constante aumento dos problemas relacionados à saúde mental dos estudantes durante a pandemia, em especial os estudantes da área da saúde, essa temática ainda vem sendo pouco discutida. Sendo um assunto pouco abordado na formação acadêmica dos estudantes de medicina, necessitando, portanto, de um maior empenho em estratégias na educação médica que desenvolvam habilidades emocionais dos discentes, além do melhor preparo dos estudantes ao lidar com o estresse durante a graduação nas escolas médicas, tendo em vista que a pandemia do COVID-19 se mostrou como um impacto estressante para os estudantes avaliados. (Felippe et al., 2021; Zonta et al., 2006).

Diante disso, faz-se necessário melhorar a rede de apoio à saúde mental dos alunos e melhor prepará-los psicologicamente ao lidar com o desconhecido, seja diante de adversidades durante o curso de medicina, seja no enfrentamento de possíveis futuras epidemias, com melhores capacitações. São necessários estudos de ensaio clínico com metodologia criteriosa sobre a temática no futuro, como pesquisas que avaliem as consequências da pandemia de COVID-19 para a formação desses acadêmicos, principalmente, a longo prazo. 


\section{Conclusão}

O presente estudo mostrou que houve um impacto estressante para os estudantes de medicina avaliados durante a pandemia do Covid-19, com potenciais prejuízos na saúde mental e qualidade de vida destes discentes. Houve associação entre percepção do risco de contágio, universidade, acompanhamento psicológico e sono em relação à escala IES de forma estatisticamente significativa. Além disso, esses dados são importantes e podem ser utilizados para futuras intervenções psicológicas, em tempos de crises como epidemias, e como base para estudos em outras escolas de ensino para diversos profissionais da saúde, como também, para avaliar, em futuras pesquisas, as repercussões da pandemia a longo prazo para estes estudantes de medicina.

\section{Referências}

Amorim, B. B., Moraes, L., Sá, I. C. G., Silva, B. B. G., \& Camara Filho, J. W. S. (2018). Saúde mental do estudante de Medicina: psicopatologia, estresse, sono e qualidade de vida. Revista Psicologia, Diversidade E Saúde, 7(2), 245-254. https://doi.org/10.17267/2317-3394rpds.v7i2.1911.

Barbosa, R. R., Martins, M. C. G., Carmo, F. P. T., Jacques, T. M., Serpa, R. G., Calil, A. O., \& Barbosa, L. F. M. (2015). Estudo sobre estilos de vida e níveis de estresse em estudantes de medicina. Int J Cardiovasc Sci, 28(4), 313-9. http://www.dx.doi.org/10.5935/2359-4802.20150045.

Brooks, S. K., Webster, R. K., Smith, L. E., Woodland, L., Wessely, S., Greenberg, N., \& Rubin, G. J. (2020). The psychological impact of quarantine and how to reduce it: rapid review of the evidence. The lancet, 395(10227), 912-920. https://doi.org/10.1016/S0140-6736(20)30460-8

Cao, W., Fang, Z., Hou, G., Han, M., Xu, X., Dong, J., \& Zheng, J. (2020). The psychological impact of the COVID-19 epidemic on college students in China. Psychiatry research, 287, 112934. https://doi.org/10.1016/j.psychres.2020.112934

Capp, E. \& Nienov, O. H. (2020). Epidemiologia aplicada básica. Porto Alegre: UFRGS.

Cardoso, F. D. A. B., Magalhães, J. F., Silva, K. M. L. D., \& Pereira, I. S. D. S. D. (2015). Perfil do estudante de Medicina da Universidade do Estado do Rio Grande do Norte (UERN), 2013. Revista Brasileira de Educação Médica, 39, 32-40. http://dx.doi.org/10.1590/1981-52712015v39n1e01092014

Deng, J., Zhou, F., Hou, W., Silver, Z., Wong, C. Y., Chang, O., ... \& Huang, E. (2021). The prevalence of depressive symptoms, anxiety symptoms and sleep disturbance in higher education students during the COVID-19 pandemic: A systematic review and meta-analysis. Psychiatry Research, 113863. https://doi.org/10.1016/j.psychres.2021.113863

Barros, G. M. M. de, Valério, F. C. E. P., Silva, M. H. F. D. da, Pecorelli, D. G., Porto , V. U. da N., \& Silva, L. de A. (2021). The impacts of the COVID-19 pandemic on the mental health of students. Research, Society and Development, 10(9), e47210918307. https://doi.org/10.33448/rsd-v10i9.18307

Felippe, T. de O., Spaniol, C. M., Silva, L. A. da ., Calabria, A. C. ., Ferreira, G. ., Carvalho, N. de L. ., Moretti, M., \& Bellinati , N. V. da C. (2021). Medical student stress during the COVID-19 pandemic. Research, Society and Development, 10(9), e58310918372. https://doi.org/10.33448/rsd-v10i9.18372

Rego, R. M., Marques, N. A., da Costa Monteiro, P., de Oliveira, C. L. B., de Almeida Lins, N. A., \& Caldas, C. A. M. (2019). O perfil atual do estudante de Medicina e sua repercussão na vivência do curso. Pará Research Medical Journal, 2(1-4), 0-0. http://dx.doi.org/10.4322/prmj.2018.005

Freitas, C. A. D., Arruda, G. F. A. D., Arruda, G. C. F. A. D., \& Feitosa, S. F. (2021). Estudantes de Medicina no enfrentamento da pandemia da Covid-19 no Brasil: reflexões éticas. Revista Brasileira de Educação Médica, 45. https://doi.org/10.1590/1981-5271v45.1-20200231

Goularte, J. F., Serafim, S. D., Colombo, R., Hogg, B., Caldieraro, M. A., \& Rosa, A. R. (2021). COVID-19 and mental health in Brazil: Psychiatric symptoms in the general population. Journal of psychiatric research, 132, 32-37. https://doi.org/10.1016/j.jpsychires.2020.09.021.

Guanilo, M. E. E. (2005). "Burns Specific Pain Anxiety Scale - BSPAS": adaptação transcultural e validação preliminar. Dissertação de Mestrado, Escola de Enfermagem de Ribeirão Preto, Universidade de São Paulo, Ribeirão Preto. doi:10.11606/D.22.2005.tde-23062005-081623 www.teses.usp.br

Horowitz, M.J., Wilner, N., \& Alvarez, W.F. (1979). Impact of Event Scale: A Measure of Subjective Stress. Psychosomatic Medicine, 41, 209-218.

Jassim, G., Jameel, M., Brennan, E., Yusuf, M., Hasan, N., \& Alwatani, Y. (2021). Psychological Impact of COVID-19, Isolation, and Quarantine: A CrossSectional Study. Neuropsychiatric disease and treatment, 17, 1413-1421. https://doi.org/10.2147/NDT.S311018

Khan, A. H., Sultana, M. S., Hossain, S., Hasan, M. T., Ahmed, H. U., \& Sikder, M. T. (2020). The impact of COVID-19 pandemic on mental health \& wellbeing among home-quarantined Bangladeshi students: a cross-sectional pilot study. Journal of affective disorders, 277, 121-128. https://doi.org/10.1016/j.jad.2020.07.135

Kubrusly, M, Coelho, RA., Augusto, KL, Peixoto Junior, AA., Santos, DC de O., \& Oliveira, CMC de. (2021). Percepção dos docentes sobre a Aprendizagem Baseada em Problemas na educação a distância durante a pandemia COVID-19. Pesquisa, Sociedade e Desenvolvimento, 10 (5), e53510515280. https://doi.org/10.33448/rsd-v10i5.15280

Ornell, F. E. L. I. P. E., Schuch, J. B., Sordi, A. O., \& Kessler, F. H. (2020). Pandemia de medo e COVID-19: impacto na saúde mental e possíveis estratégicas. Revista debates in psychiatry, 2020.

Odriozola-González, P., Planchuelo-Gómez, Á., Irurtia, M. J., \& de Luis-García, R. (2020). Psychological effects of the COVID-19 outbreak and lockdown among students and workers of a Spanish university. Psychiatry research, 290, 113108. https://doi.org/10.1016/j.psychres.2020.113108 
Research, Society and Development, v. 11, n. 3, e10811326341, 2022

(CC BY 4.0) | ISSN 2525-3409 | DOI: http://dx.doi.org/10.33448/rsd-v11i3.26341

Rose S. (2020). Medical Student Education in the Time of COVID-19. JAMA, 323(21), 2131-2132. https://doi.org/10.1001/jama.2020.5227

Prashanth, N. T., Raghuveer, H. P., Kumar, R. D., Shobha, E. S., Rangan, V., \& Hullale, B. (2015). Post-traumatic Stress Disorder in Facial Injuries: A Comparative Study. The journal of contemporary dental practice, 16(2), 118-125. https://doi.org/10.5005/jp-journals-10024-1647

Silva, A. C. D. O., Nardi, A. E., \& Horowitz, M. (2010). Brazilian version of the Impact of Event Scale (IES): translation and cross-cultural adaptation. Revista de Psiquiatria do Rio Grande do Sul, 32(3), 86-93. https://doi.org/10.1590/S0101-81082010000300005

Song, Y., Bai, W., Wang, M., Liu, X., Zhang, L., Yu, W., Li, Y., Hua, W., Lew, B., Talib, M.A., \& Kou, C. (2019). The association between psychological strain and suicidal behaviors among college students: A mental health survey in Jilin Province, Northeast China. Journal of affective disorders, 259, 195-200. https://doi.org/10.1016/j.jad.2019.08.042

Taghrir, M. H., Borazjani, R., \& Shiraly, R. (2020). COVID-19 and Iranian Medical Students; A Survey on Their Related-Knowledge, Preventive Behaviors and Risk Perception. Archives of Iranian medicine, 23(4), 249-254. https://doi.org/10.34172/aim.2020.06

Tee, M. L., Tee, C. A., Anlacan, J. P., Aligam, K., Reyes, P., Kuruchittham, V., \& Ho, R. C. (2020). Psychological impact of COVID-19 pandemic in the Philippines. Journal of affective disorders, 277, 379-391. https://doi.org/10.1016/j.jad.2020.08.043

Teixeira, L. D. A. C., Costa, R. A., Mattos, R. M. P. R. D., \& Pimentel, D. (2021). Saúde mental dos estudantes de Medicina do Brasil durante a pandemia da coronavirus disease 2019. Jornal Brasileiro de Psiquiatria, 70, 21-29. https://doi.org/10.1590/0047-2085000000315

Tolsgaard, M. G., Cleland, J., Wilkinson, T., \& Ellaway, R. H. (2020). How we make choices and sacrifices in medical education during the COVID-19 pandemic. Medical teacher, 42(7), 741-743. https://doi.org/10.1080/0142159X.2020.1767769

Torun, F., \& Torun, S. D. (2020). The psychological impact of the COVID-19 pandemic on medical students in Turkey. Pakistan journal of medical sciences, $36(6), 1355-1359$.

Vanaken, L., Scheveneels, S., Belmans, E., \& Hermans, D. (2020). Validation of the Impact of Event Scale with Modifications for COVID-19 (IESCOVID19). Frontiers in psychiatry, 11, 738.

Wang, C., Pan, R., Wan, X., Tan, Y., Xu, L., Ho, C. S., \& Ho, R. C. (2020). Immediate Psychological Responses and Associated Factors during the Initial Stage of the 2019 Coronavirus Disease (COVID-19) Epidemic among the General Population in China. International journal of environmental research and public health, 17(5), 1729.

Zhang, Y., \& Ma, Z. F. (2020). Impact of the COVID-19 Pandemic on Mental Health and Quality of Life among Local Residents in Liaoning Province, China: A Cross-Sectional Study. International journal of environmental research and public health, 17(7), 2381.

Zonta, R., Robles, A. C. C., \& Grosseman, S. (2006). Estratégias de enfrentamento do estresse desenvolvidas por estudantes de medicina da Universidade Federal de Santa Catarina. Revista Brasileira de Educação Médica, 30, 147-153. 